\title{
THE STORY OF A STONE HEART - ANOTHER SIDE OF CARDIOONCOLOGY
}

\author{
Maia Rusu', Tudor Constantinescu, ${ }^{2,3}$, Ruxandra Jurcuţ ${ }^{1,3}$ \\ ${ }^{1}$ Institutul de Urgenţă pentru Boli Cardiovasculare "Prof. dr. C. C. Iliescu", Bucureşti \\ 2Spitalul de Pneumoftiziologie "Marius Nasta", Bucureşti \\ "UMF "Carol Davila", Bucureşti
}

Keywords: pulmonary sarcoma, thoracic radiotherapy, cardiac calcifications.

A 38-year-old man was admitted to our department with moderate exertional dyspnea, fatigue and a syncope during exercise. The medical history revealed: left pulmonary sarcoma at the age of four, treated with radiotherapy, chemotherapy and left total pneumonectomy. At admission, laboratory tests showed high BNP (1426 pg/ml), normal calcium and parathormon levels. The transthoracic echocardiography found normal left ventricular (LV) systolic function with severe calcifications of the papillary muscles, mitral annulus, apical segments of the inferior septum and inferior wall (panel A - arrows; panel D) associated with severe mitral regurgitation (panel $B$ ). It also revealed severe tricuspid valve regurgitation, severe pulmonary hypertension (estimated to $120 \mathrm{mmHg}$, panel C) and small amount of pericardial fluid. A thoracic computed tomography described severe cardiac calcifications (panels E and F, arrow), a hypertrophic right lung herniated in left hemythorax and no other pathological findings in the remnant lung tissue. The pneumological evaluation noted a severe restrictive dysfunction. In this case, pulmonary hypertension was most probably determined by the left cardiac disorders (severe mitral regurgitation postradiotherapy, LV diastolic dysfunction due to severe myocardial and papillary muscle calcifications), most probably related to thoracic radiotherapy during childhood. 


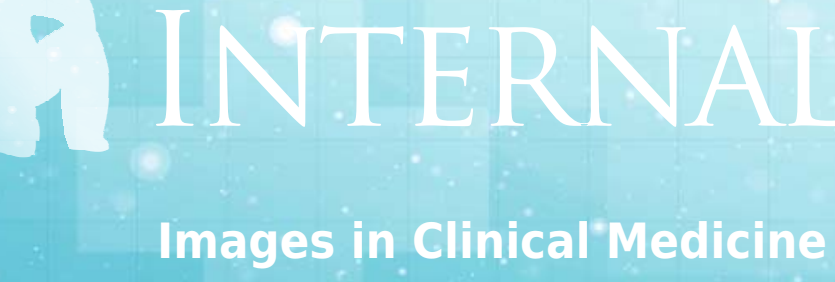
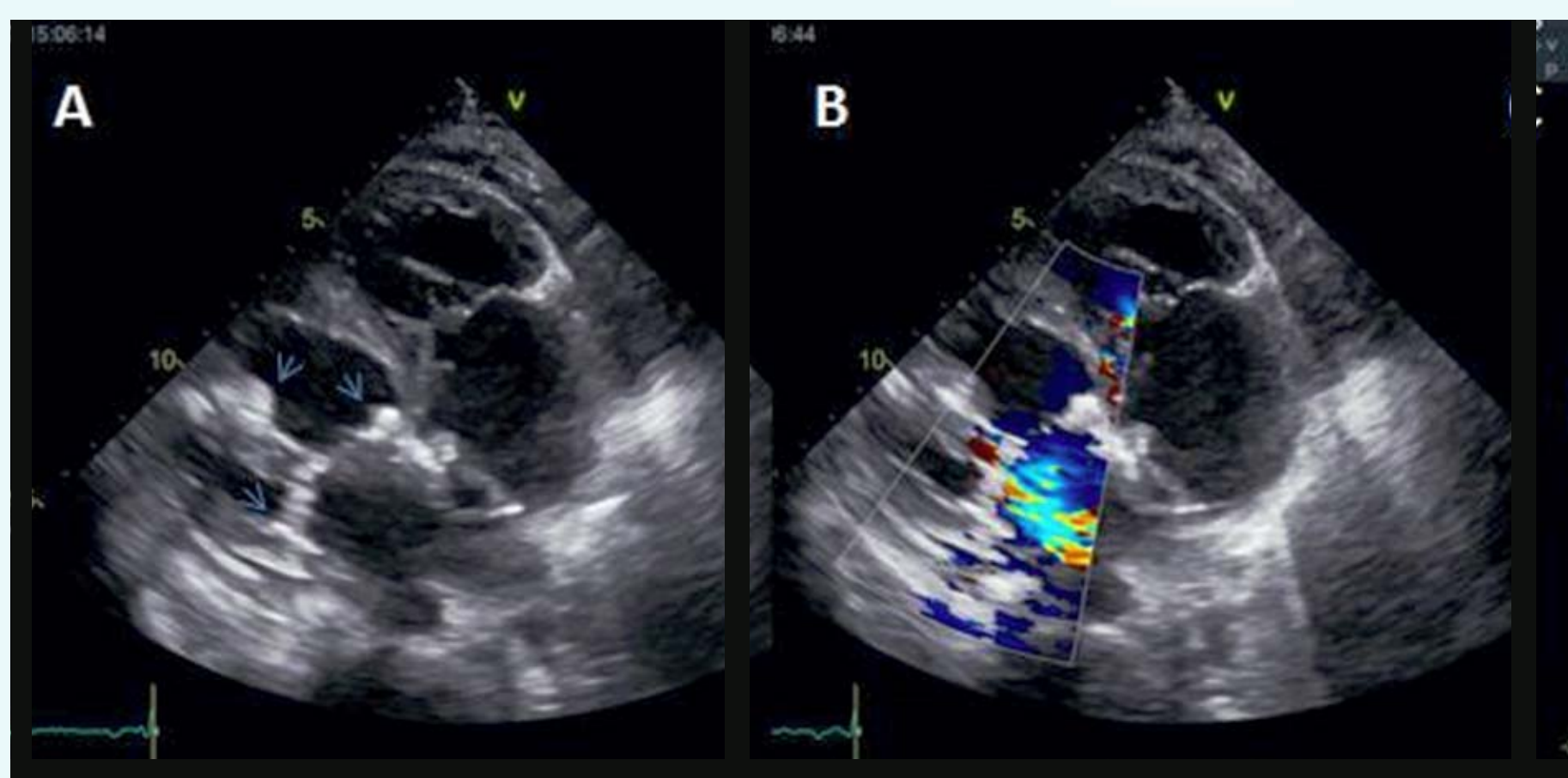

\section{D}
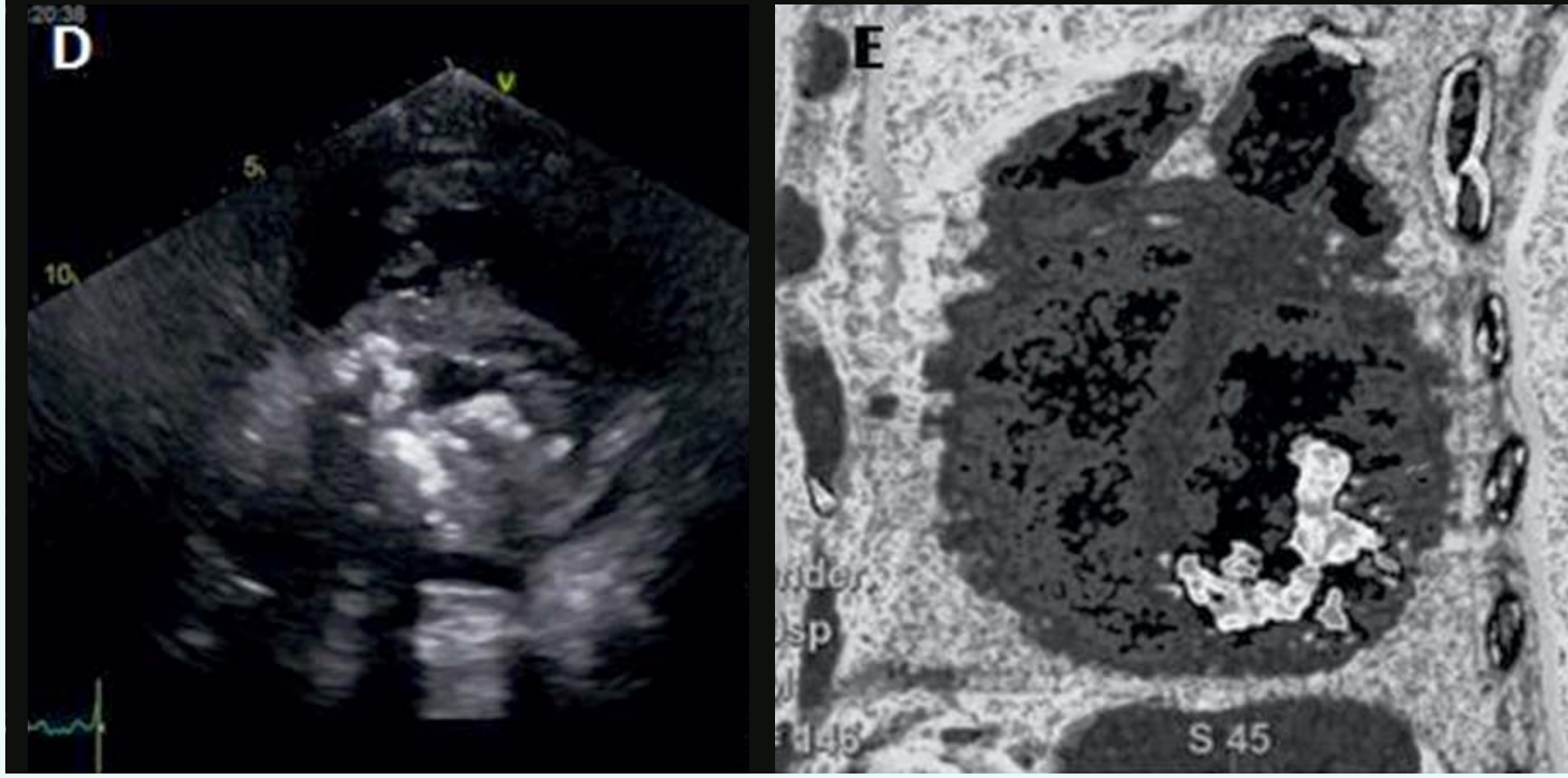


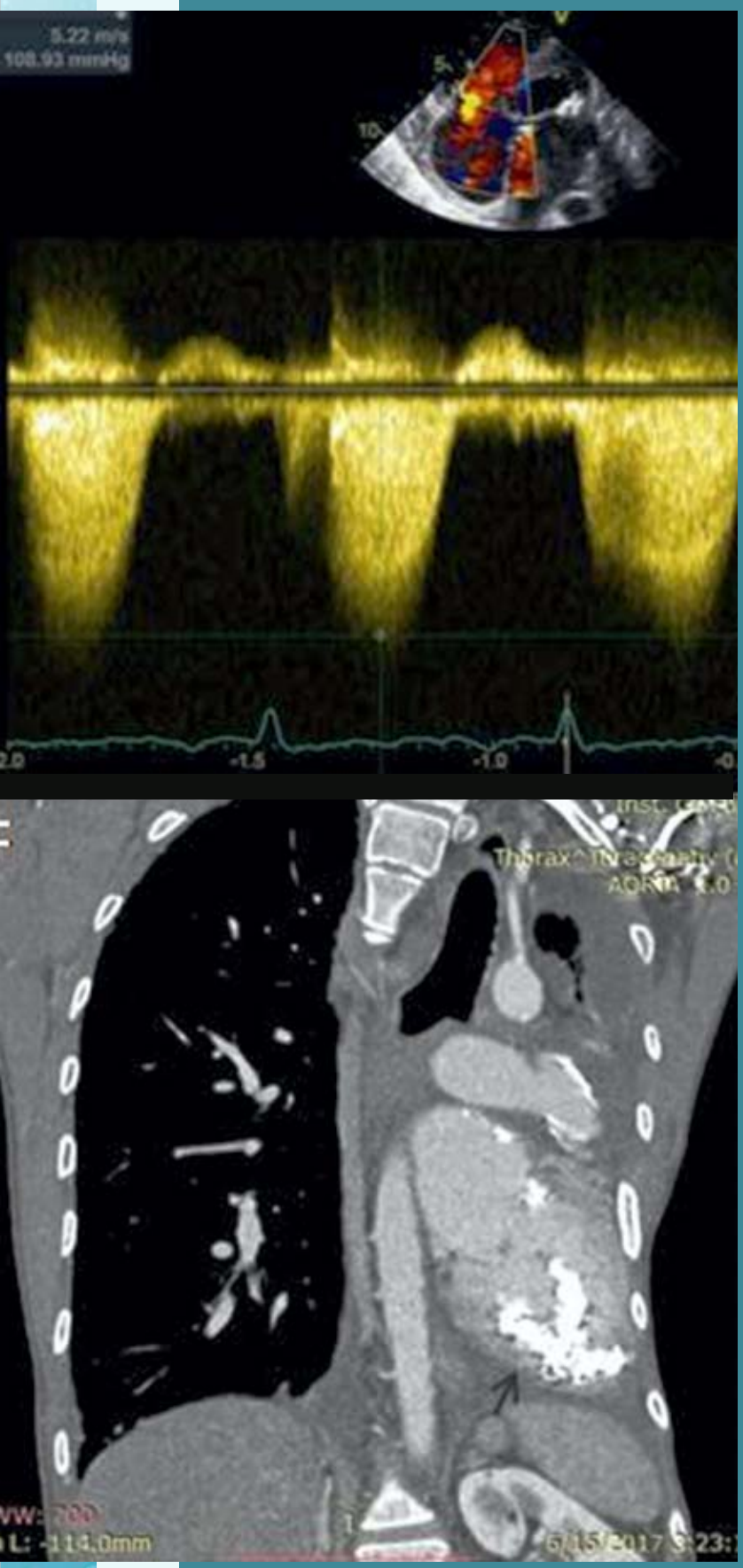

Figure 1. Transthoracic

echocardiography - severe

calcifications of the papillary muscles, mitral annulus, apical segments of the inferior septum and inferior wall (panel A - arrows; panel D), severe mitral regurgitation (panel $B$ ), tricuspid valve regurgitation, severe pulmonary hypertension (panel C). Thoracic computed tomography severe cardiac calcifications (panels E and $F$, arrow), hypertrophic right lung herniated in left hemythorax.

Aknowledgements: The authors thank Teodora Barascu for technical assistance with CT image processing. 